\title{
Hobbits, Hogwarts, and the Heavens: The use of fantasy literature and film in astronomy outreach and education
}

\author{
Kristine Larsen \\ Physics and Earth Sciences, Central Connecticut State University, \\ 506 Copernicus Hall, 1615 Stanley St., New Britain, CT 06050, USA \\ email: larsen@ccsu.edu
}

\begin{abstract}
Due in part to recent (and ongoing) film adaptations, the fantasy series of C.S. Lewis (The Chronicles of Narnia), J.K. Rowling (Harry Potter), Philip Pullman (His Dark Materials), and J.R.R. Tolkien (The Silmarillion, The Hobbit, and The Lord of the Rings) are being introduced to a new audience. Many astronomers and astronomy educators are unaware of the wide variety of astronomical references contained in each series, and the myriad possible uses of these works in astronomy education and outreach. This paper highlights activities which educators, planetariums, and science centers have already developed to utilise these works in their education and outreach programs.
\end{abstract}

Keywords. Science fiction and fantasy, popular culture, literature, film

In his classic essay, On Fairy-Stories, philologist and fantasy writer J.R.R. Tolkien (Tolkien 1997) argued that fantasy "does not destroy or even insult Reason; and it does not either blunt the appetite for, nor obscure the perception of scientific verity. On the contrary, the keener and clearer the reason, the better the fantasy it will make". Although literary critics have argued for a distinction between fantasy and science fiction, with only the latter considered to be based on scientific principles, in the case of four specific fantasy series -those of C.S. Lewis (The Chronicles of Narnia), J.K. Rowling (Harry Potter), Philip Pullman (His Dark Materials), and J.R.R. Tolkien (The Silmarillion, The Hobbit, and The Lord of the Rings), the distinction is significantly blurred. Regardless of the specific classification used, the works of all four authors can and have been successfully used in astronomy education and outreach, as described in this paper.

Considered the archetype of its literary class, J.R.R. Tolkien's sweeping fantasy series centered on Middle-earth consists of The Silmarillion (1977), The Hobbit (1937), and The Lord of the Rings (1955), as well as a number of posthumously published works (edited by his son, Christopher), including twelve detailed volumes of drafts and alternate texts referred to as the History of Middle-Earth series. Each of these works draws heavily on Tolkien's academic interests in language, culture, mythology, and science, and contain an impressive litany of astronomical knowledge, reflecting Tolkien's childhood interest in astronomy and other sciences (Tolkien 1997). Astronomical references in the works of Tolkien include constellations, a detailed creation myth, mythical explanations for eclipses and lunar phases, the Morning Star, auroras, the use of meteoritic iron in weaponry, the development of several precise calendars, and the use of lunar phases to coordinate the chronology of events in The Lord of the Rings. Detailed descriptions of astronomical references in Tolkien's works can be found in Quiñonez \& Raggett (1990) and Larsen (2005,b, 2006, 2007, 2009). The Lord of the Rings has consistently topped a number of readers' polls conducted in several countries over the past decade, and the 
movies based on the trilogy broke sales records around the world. Two movies based on The Hobbit are due to be released in 2012 and 2013.

One of Tolkien's closest friends was C.S. Lewis, and the pair belonged to a small literary/social circle known as The Inklings. Lewis was an amateur astronomer and used both his own small telescope and the Oxford University Observatory (Ward 2008). The Chronicles of Narnia series is comprised of seven books published between 1950-1956 which documents the parallel universe of Narnia from its creation to its destruction. Characters include stars who have retired from the sky and star-gazing dwarfs and centaurs, and topics such as constellations, meteors, planetary alignments, the Evening Star, and the deaths of stars find a place in the Narnian cosmology. The series has sold over 100 million copies worldwide, and the second movie based on the series was released in 2008, with a third to follow in 2010.

Phillip Pullman describes science as a topic that he was "fascinated by at home and turned off by at school" (Gribbin \& Gribbin 2007). His Subtle Materials trilogy, comprised of The Golden Compass, The Subtle Knife, and The Amber Spyglass (1995-2000), is filled with philosophical debates and an exploration of the tension between fundamentalist dogma and scientific exploration. Main character Lyra Belacqua travels between parallel universes, and in the process the reader learns much about aurora, dark matter, and evolution. Iorek Byrnison, the much-beloved king of the polar bears, makes his own armor out of meteoritic iron. The series has won several prestigious awards, and came in third behind The Lord of the Rings and Jane Austen in a 2003 BBC readers poll. The first book has already been made into a film (2007), and there has been discussion of adapting the second book for the big screen.

No recent fantasy series has sold as many copies as those of J.K. Rowling's Harry Potter universe (or 'Potterverse'). The seven novels have broken all sales records, and movies based on the first five books have opened to extremely large and enthusiastic crowds. Harry Potter and the Half-Blood Prince is due to open in theaters in July 2009, with two movies based on the final book due to open in 2011 and 2012. As with the other authors discussed, Rowling has interwoven a great deal of astronomy into her series. In book five, Harry Potter and The Order of the Phoenix, the young protagonists study the Galilean moons of Jupiter in preparation for the astronomy portion of their Ordinary Wizardry Level (OWL) exam, which entails an observational astronomy practicum using a telescope. Numerous characters sport astronomical names, such as Sirius Black, Andromeda Tonks, and Merope Gaunt, further introducing Rowling's young readers to astronomical concepts and terminology. Astronomical references in the Potterverse are explained in detail in James (2007), Krisciunas (2003), and Pasachoff (2003).

Therefore, just as numerous authors have successfully utilized works of science fiction in astronomy and physics education and outreach (e.g.. Allday 2003; Barnett \& Kafka 2007; Dubeck 1994), these and other fantasy series can act as valuable partners in our astronomy education and outreach toolkits, and should be exploited often and shamelessly in order to interest the general public (and college students) in astronomy. Given the plethora of astronomical references in these works, astronomy educators have a wide variety of astronomical concepts which can be illustrated. For example, a lecture on auroras can be introduced with one of Pullman's beautifully descriptive passages on the Northern Lights from The Golden Compass (e.g.. Pullman 2007). Similarly, citations from these works can be used on exams and other assessments to measure students' ability to apply their astronomical knowledge to interdisciplinary situations. For example, students can apply their understanding of the end stages of stellar evolution to passages from Lewis's The Magician's Nephew which describes a dying world orbiting a red giant star (e.g., 
Lewis 2008). Students can also relate the ancient and medieval concept of the music of the spheres to the musical modes of creation of the universes of Middle-earth and Narnia.

Since both the tales of Narnia and Middle-Earth contain detailed creation myths, these stories can be used as concrete examples for the analysis of creation myths, without the worry of offending students of any ethnic or religious background. Students can further apply their understanding of the function and format of creation myths by writing their own creation myth for an alien world with parameters set by the instructor. An example assignment can be found at http://www.physics.ccsu.edu/larsen/paper_1.htm.

Lab exercises can also be developed around the astronomical references found in these works. For example, the lunar chronology of The Lord of the Rings is the basis of an exercise entitled What Day is it, Dr. Frodo?, while the constellations of Middle-Earth are the central focus of The Stars of Middle-Earth. Both exercises appear in the Astronomical Society of the Pacific's volume Cosmos in the Classroom 2004 (Larsen 2004).

The most extreme case is, of course, the development of an entire course focusing on the astronomy or other sciences found in these works. An example is The Science of Middle-Earth, a course for first-year students not majoring in the sciences. The course was taught as part of a learning community with a section of Freshman Composition. The same cohort of students was registered for both sections, and read The Hobbit, The Lord of the Rings, The Silmarillion, and selections from Tolkien's letters and the History of Middle-Earth volumes. Among the scientific topics covered were science and religion, ancient astronomy, the night sky, calendars, star birth and death, formation of the earth and moon, plate tectonics, volcanoes, meteorites, the origin of life, evolution, and genetic engineering. A course syllabus and other materials can be found at http://www.physics.ccsu.edu/larsen/courses_fall_2007.htm.

While astronomy education certainly affords opportunities for the creative use of fantasy literature and film, outreach activities are more flexible in terms of time, content, and audience. Therefore, fantasy series are even more valuable to the astronomy outreach specialist. An obvious example is planetarium shows and star parties, the staples of astronomy outreach. Harry Potter themes have been successfully utilized in star parties by the Jodrell Banks Observatory Visitors Center and other organisations, especially when such events have been tied in with the release of books in the series (http://www.strudel.org.uk/blog/astro/000676.shtml).

The December 2008 Venus-Jupiter conjunction also afforded a natural tie-in with the Potterverse (Larsen 2008), given the inclusion of the Galilean satellites, planetary alignments, and viewing Venus in a telescope in the Hogwarts OWLS exam. Visitors to the observing sessions at Central Connecticut State University's Copernican Observatory received a special OWLS exam certificate. NASA also used the OWLS astronomy exam as the basis for a feature article on the Galilean moons (http://www.nasa.gov/vision/ universe/solarsystem/02jul_harrypotter.html). The New Jersey Center for Education has developed a Harry Potter planetarium show, The Skies Over Hogwarts based on astronomical themes and characters in the series (http://www.raritanval.edu/ planetarium/planetarium.htm). The classic starwheel activity done in numerous classrooms and astronomy workshops can easily be adapted to either Middle-Earth or the Potterverse. For example, Chapter III of The Fellowship of the Ring contains the following detailed description of the midnight sky in late September:

Away high in the East swung Remmirath, the Netted Stars, and slowly above the mists red Borgil rose, glowing like a jewel of fire. Then by some shifts of airs all the mist was drawn away like a veil, and there leaned up, as he climbed over the rim of the world, the Swordsman of the Sky, Menelvagor with his shining belt. 
Remmirath is the Pleiades, Borgil is Aldebaran, and Menelvagor is most obviously Orion. Turning to the Potterverse, fans can be enticed to study the constellations by turning the classic free online planisphere 'Uncle Al's Skywheel' (available at http://www. Ihs . berkeley.edu/StarClock/skywheel.html) into a Harry Potter Starfinder by simply labeling Alphard and Bellatrix, and coupling it with a genealogy of the Black family (e.g. http://www.hp-lexicon.org/wizards/blackfamilytree.html). Rowling's chief astronomical error (having the Hogwarts students view Orion before midnight in June) can easily be illustrated using this planisphere. The activity can be tied to a planetarium show focusing on the constellations, or with an activity in which flashcards are made for each of the relevant constellations and stars (e.g. http://www.ccsu.edu/astronomy/ SiriusBlack.docx).

As mentioned previously, the Galilean moons of Jupiter are described in Harry Potter and the Order of the Phoenix as part of astronomy professor Aurora Sinistra's dreaded essay. The characteristics of these moons can be explored in a number of hands-on activities, such as making scale models of Jupiter and the Galilean moons and the Earth-Moon system using paper and string. A useful scale is to make Earth approximately three inches wide. In this model, a quadrant of Jupiter is roughly the size of a full-size sheet of newspaper.

Centaurs figure prominently in the Potterverse, and play the rôle of astronomers (or at least stargazers). This connection can be used to introduce children and adults to Sagittarius as a constellation, and the myriad astronomical objects contained within its borders. Among these is notably the center of our galaxy. The galaxy is mentioned in Harry Potter and the Prisoner of Azkaban, when Harry contemplates purchasing a moving model on a shopping trip to Diagon Alley. The structure of the galaxy can be discussed in this context, using an extension of the 'glitter galaxy' (http://www . enchantedlearning . com/crafts/astronomy/glittergalaxy). Instead of using actual glitter, glitter glue pens of the appropriate colors (red and blue for the spiral arms and yellow for the central region) cut down considerably on the mess, and globular clusters can be depicted by tiny pieces torn from cotton balls and glued to the paper.

Numerous authors have successfully utilised science fiction works such as Star Trek and short stories by Isaac Asimov as tools in teaching science since the 1950s. Classic fantasy series, such as those by Tolkien, Lewis, Pullman, and Rowling are beginning to take their rightful place in this tradition. As explained in this paper, the astronomical references are there for the taking, and the only limitation to their use in astronomy outreach and teaching is the creativity of the astronomical community. For additional examples and resources, the interested reader is directed to http://www.ccsu.edu/astronomy/ hobbits.htm.

\section{References}

Allday, J. 2003, Physics Education, 38, 27

Barnett, M. \& Kafka, A. 2007, J. College Sci. Teaching, 36, 31

Dubeck, L. W., Mosher, S. E., \& Boss, J. E. 1994, Fantastic Voyages: Learning Science Through Science Fiction Films (Woodbury, NY: AIP Press)

Gribbin, M. \& Gribbin, J. 2007, The Science of Philip Pullman's His Dark Materials (New York: Laurel-Leaf)

James, C. R. 2007, Mercury, 36, 20

Krisciunas, K. 2003, Sky \& Telescope, 106, 12

Larsen, K. 2004, in Cosmos in the Classroom 2004, A. Fraknoi \& W. Waller (eds) (San Francisco: Astronomical Society of the Pacific), p. 237

Larsen, K. 2005a, Tolkien Studies, 2, 161 
Larsen, K. 2005b, Mallorn, 43, 49

Larsen, K. 2006, Mallorn, 44, 22

Larsen, K. 2007, Tolkien Studies, 4, 223

Larsen, K. 2008, CAP Journal, 4, 16

Larsen, K. 2009, in The Mirror Crack'd: Fear and Horror in J. R. R. Tolkien's The Lord of the Rings and its Sources L. Forest-Hill (ed) (Cambridge: Cambridge Scholars Publishing), p. 169

Lewis, C. S. 2008, The Chronicles of Narnia (New York: Harper Collins)

Pasachoff, J. M. 2003, Sky \& Telescope, 106, 12

Pullman, P. 2007, His Dark Materials (New York: Alfred A. Knopf)

Quiñonez, J. \& Raggett, N. 1990, Vinyar Tengwar, 12, 5

Tolkien, J. R. R. 1994, The Lord of the Rings (Boston: Houghton Mifflin)

Tolkien, J. R. R. 1997, The Monsters and the Critics (London: Harper Collins)

Ward, M. 2008, Planet Narnia: The Seven Heavens in the Imagination of C. S. Lewis (Oxford: Oxford University Press) 